\title{
Nematoides anisaquídeos e cestoides Trypanorhyncha de importância em saúde pública em Aluterus monoceros (Linnaeus, 1758) no Estado do Rio de Janeiro, Brasil
}

\author{
Larvae of Anisakidae nematodes and Trypanorhyncha cestodes of public health \\ importance in Aluterus monoceros (Linnaeus, 1758) in Rio de Janeiro State, Brazil \\ Fátima de Jesus Esteves Dias ${ }^{1}$; Sérgio Carmona de São Clemente ${ }^{1 *}$; Marcelo Knoff ${ }^{2}$
}

${ }^{1}$ Faculdade de Medicina Veterinária, Universidade Federal Fluminense - UFF

${ }^{2}$ Laboratório de Helmintos Parasitos de Vertebrados, Instituto Oswaldo Cruz - FIOCRUZ

Recebido em 10 de Agosto de 2009

Aceito em 20 de Agosto de 2009

\section{Resumo}

Entre maio e agosto de 2006, foram adquiridos 100 espécimes de peixe-porco, Aluterus monoceros em estabelecimentos de pescado nos municípios de Niterói e Rio de Janeiro. Os peixes foram medidos, necropsiados, filetados e seus órgãos analisados. Foram encontrados 16 peixes parasitados por larvas de nematoides anisaquídeos pertencentes às espécies Anisakis sp. e Contracaecum sp., com prevalência de 1 e 16\%, intensidade média de 2 e 3,31 e abundância média de 0,02 e 0,53, respectivamente. Duas larvas de Anisakis sp. foram encontradas no mesentério de um peixe; e de Contracaecum sp. no fígado e mesentério, com amplitude de variação da intensidade de infecção de 1 a 9 . Cinquenta e um peixes mostravam-se parasitados no fígado e mesentério por cestoides da ordem Trypanorhyncha. As espécies colhidas foram Floriceps saccatus e a Callitetrarhynchus speciosus, com a prevalência de 45 e 6\%, intensidade média de 3,17 e 2,83, variando de 1 a 20 e 1 a 5, e abundância média de 1,43 e 0,06, respectivamente. Larvas de Anisakis sp. e essas duas espécies de Trypanorhyncha foram registradas pela primeira vez parasitando $A$. monoceros.

Palavras-chave: Anisakis sp., Contracaeum sp., Floriceps saccatus, Callitetrarhynchus speciosus, Aluterus monoceros.

\begin{abstract}
One hundred specimens of unicorn leatherjacket, Aluterus monoceros purchased from markets of municipalities of Niterói and Rio de Janeiro from May to August 2006. The fishes were measured, necropsied, fileted and analysed their organs. Sixteen fishes were parasitized by nematode Anisakidae: Anisakis spp. and Contracaecum sp. with respectively, 1 and $16 \%$ of prevalence, 2 and 3.31 of mean intensity, and 0.02 and 0.53 of mean abundance. Two larvae of Anisakis sp. were found in mesentery of one fish and Contracaecum sp. was found in liver and mesentery with 1 to 9 specimens of range of infection. Fifty-one fishes were parasitized on the liver and mesentery by metacestodes of Trypanorhyncha. The collected species were Floriceps saccatus and Callitetrarhynchus speciosus with respectively, 45 and 6\% of prevalence, 3.17 and 2.83 of mean intensity, and 1.43 and 0.06 of mean abundance, the range of infection by $F$. saccatus was 1 to 20 and by $C$. speciosus was 1 to 5 . Anisakis sp. and these two species of Trypanothyncha were reported in A. monoceros for the first time.
\end{abstract}

Keywords: Anisakis sp., Contracaeum sp., Floriceps saccatus, Callitetrarhynchus speciosus, Aluterus monoceros.

\section{Introduçáo}

A espécie Aluterus monoceros, conhecida como peixe-porco, tem distribuição praticamente cosmopolita. No Atlântico Ocidental, estende-se da Nova Inglaterra, no Canadá, até a Argentina (FIGUEIREDO; MENEZES, 2000).

A presença de parasitos nos produtos da pesca constitui perigo sanitário que não deve ser subestimado. Ainda que a maioria desses

\footnotetext{
*Autor para correspondência: Sérgio Carmona São Clemente

Faculdade de Medicina Veterinária, Universidade Federal Fluminense - UFF,

Rua Vital Brazil Filho, 64, Vital Brazil, CEP 24.230-340, Niterói - RJ, Brasil;

e-mail: scsc@vm.uff.br
}

organismos náo seja patogênica para o homem, algumas espécies podem originar enfermidades graves em virtude da ingestáo de pescado parasitado, sendo a mais conhecida aquela veiculada por larvas de nematoides da família Anisakidae (GONZÁLEZ, 2003).

As infecçóes humanas por esses parasitos estão associadas ao consumo de pescados crus ou submetidos a processos que não alteram a viabilidade das larvas, como é o caso da ingestão de pescado cru ou com pouca cocção (FERRE, 2001).

De acordo com Audicana et al. (2000), as afecçóes do trato gastrintestinal de humanos, durante a infecção por larvas 
de Anisakis simplex, são resultado da ação traumática pela invasão tecidual e pela interação com o sistema imunitário do hospedeiro e o conjunto de substâncias liberadas ou contidas no parasito. Na maioria dos casos, é produzida por uma única larva e, embora mais frequente no trato digestório, algumas larvas podem atravessar a parede gastrintestinal, migrando para localizações ectópicas.

A ocorrência de larvas de anisaquídeos, que parasitam peixes marinhos do litoral do Estado do Rio de Janeiro, foi registrada por Knoff et al. (2007). Embora no Brasil não existam, até o presente momento, relatos sobre anisaquiose humana esta pode tornar-se uma zoonose emergente no país (GERMANO, P.M.L.; GERMANO, M.I.S., 1998).

A análise visual dos peixes, descartando-se espécimes parasitados, é recomendada pela legislação de diversos países, como Brasil e Espanha, entre outros (BRASIL, 2007).

Os cestoides Trypanorhyncha são representados por grande diversidade de espécies que parasitam peixes e invertebrados marinhos. Os vermes adultos são encontrados no intestino de elasmobrânquios, e larvas na cavidade celomática e musculatura de teleósteos, crustáceos e moluscos cefalópodes (CAMPBELL; BEVERIDGE, 1994).

Os Trypanorhyncha adquirem importância pelo aspecto repugnante que conferem ao pescado, motivo de descarte na indústria de beneficiamento ou nos serviços de inspeção. São Clemente et al. (2007) registraram o parasitismo por cestoides Trypanorhyncha em peixes marinhos do litoral do Estado do Rio de Janeiro.

Cistos de Trypanorhyncha não são transmissíveis aos vertebrados homeotérmicos, e um novo encapsular das pós-larvas não ocorre em animais de sangue quente (DOLLFUS, 1942). Porém, larvas de Trypanorhyncha localizadas na musculatura dos peixes podem produzir toxinas e afetar o consumidor (DEARDORFF et al., 1984). Alguns registros demonstram que extratos de determinada espécie de Trypanorhyncha induzem respostas humorais em camundongos, suscitando a possibilidade de reaçôes alérgicas em humanos (RODERO; CUÉLLAR, 1999; VÁZQUEZ-LÓPEZ et al., 2002).

Este trabalho teve por objetivo o estudo de larvas de nematoides Anisakidae e de plerocercos de cestoides Trypanorhyncha, presentes em Aluterus monoceros, comercializados nos municípios de Niterói e Rio de Janeiro, estabelecendo seus índices parasitários e sítios de infecção, o que enfatiza sua importância na inspeção sanitária e saúde pública.

\section{Material e Métodos}

De maio a agosto de 2006, foram adquiridos 100 espécimes de Aluterus monoceros, em mercados de pescado dos municípios de Niterói e Rio de Janeiro. Os peixes mediam entre 44,1 e 68,0 cm $(\overline{\mathrm{x}}=56,05 \mathrm{~cm})$ de comprimento total (CT) e foram divididos em quatro classes de tamanho: $A=44,1$ a $50,0 \mathrm{~cm}, \mathrm{~B}=50,1 \mathrm{a} 56 \mathrm{~cm}$, $\mathrm{C}=56,1$ a $62,0 \mathrm{~cm}$ e $\mathrm{D}=62,1$ a $68,0 \mathrm{~cm}$. Os peixes foram identificados conforme Figueiredo e Menezes (2000).

Após a coleta, os peixes foram transportados em recipientes isotérmicos, contendo gelo, para o Laboratório de Inspeção e Tecnologia de Pescado da Faculdade de Veterinária da Universidade Federal Fluminense, onde foram identificados, medidos, necropsiados e filetados. Os helmintos encontrados foram colocados em placas de Petri, contendo solução $\mathrm{NaCl}$ a $0,65 \%$. Os nematoides foram fixados em AFA à quente $\left(60^{\circ} \mathrm{C}\right)$, preservados em álcool $70^{\circ} \mathrm{GL}$, glicerinado a $5 \%$ e clarificados com lactofenol de Aman. Os plerocercos (larvas de cestoides formadoras de blastocistos) de Trypanorhyncha foram transferidos para água destilada e tiveram seus cistos rompidos com o auxílio de estiletes para liberação sob o estereomicroscópio. Em seguida, foram transferidos para o refrigerador, por no mínimo 24 horas, possibilitando o relaxamento dos escólices e extroversão dos tentáculos. Posteriormente, as larvas foram fixadas em AFA, coradas pelo carmim de Langeron, diferenciadas em álcool clorídrico a $0,5 \%$, desidratadas em série alcoólica crescente, clarificadas em creosoto de Faia e montadas entre lâmina e lamínula com bálsamo do Canadá ou preservadas em álcool $70^{\circ} \mathrm{GL}$. Os procedimentos de necropsia dos peixes e o processamento dos helmintos foram realizados de acordo com Eiras, Takemoto e Pavanelli (2006).

A determinaçáo taxonômica dos gêneros das larvas de nematoides Anisakidae teve como base os trabalhos de Rego et al. (1983) e Petter e Maillard (1988), assim como a dos cestoides Trypanorhyncha foi realizada, segundo Carvajal e Rego (1985) e Campbell e Beveridge (1994).

As terminologias de ecologia parasitária seguem o proposto por Bush et al. (1997). O teste Qui-quadrado foi aplicado para verificar a correlação entre as classes de tamanho e o número de peixes parasitados. Espécimes representativos dos parasitos foram depositados na Coleção Helmintológica do Instituto Oswaldo Cruz (CHIOC).

\section{Resultados}

Dos 100 peixes $A$. monoceros coletados, $16 \%$ apresentaram nematoides da família Anisakidae: Anisakis sp. e Contracaecum sp.; e $51 \%$ dos peixes estavam parasitados por plerocercos da Ordem Trypanorhyncha: Floriceps saccatus Cuvier, 1817 e Callitetrarhynchus speciosus (Linton, 1897). Os índices parasitários de prevalência, intensidade média, amplitude de variação da intensidade e abundância média de infecção, bem como os sítios de infecção e o número de depósito na $\mathrm{CHIOC}$ estão apresentados na Tabela 1. Os índices parasitários das larvas de Anisakidae e dos plerocercos de Trypanorhyncha, em relação aos intervalos de classe do comprimento total dos hospedeiros, estáo apresentados na Tabela 2 e Tabela 3, respectivamente.

Aplicando-se o Teste Qui-quadrado não foi observado diferença significativa entre as classes de tamanho e o número de peixes parasitados em relação à presença das larvas de Anisakidae e Trypanorhyncha.

Este é o primeiro registro das espécies Anisakis sp., Floriceps saccatus e Callitetrarhynchus speciosus em Aluterus monoceros.

\section{Discussão}

$\mathrm{Na}$ presente pesquisa, foi observada a correlação positiva entre tamanho de espécimes e parasitismo por nematoides, com prevalência de $9 \%$ por Contracaecum sp. na classe de maior tamanho, além da coleta de Anisakis sp. em um peixe também dessa classe de tamanho, embora a prevalência citada na classe B tenha sido maior que a da classe C. Esse fato pode ser atribuído à dinâmica 
Tabela 1. Prevalência (P), intensidade e intensidade média (I/IM), abundância média (AM), amplitude de variação da intensidade de infecção (AI), sítio de infecção e o número do depósito na CHIOC das larvas de nematoides Anisakidae e dos plerocercos de cestoides Trypanorhyncha, coletados de Aluterus monoceros, comercializados no estado do Rio de Janeiro, no período de maio a agosto de 2006.

\begin{tabular}{|c|c|c|c|c|c|c|}
\hline Espécies de helmintos & $\mathbf{P}(\%)$ & I*/IM & AM & AI & Sítio de infecçáo & No CHIOC \\
\hline Anisakis sp. & 1 & $2^{*}$ & 0,02 & - & mesentério & 35638 \\
\hline Contracaecum sp. & 16 & 3,31 & 0,53 & $1-9$ & fígado e mesentério & 35639 \\
\hline Floriceps saccatus & 45 & 3,17 & 1,43 & $1-20$ & fígado e mesentério & 35636 \\
\hline Callitetrarhynchus speciosus & 6 & 2,83 & 0,06 & $1-5$ & fígado e mesentério & 35637 \\
\hline
\end{tabular}

*apenas um peixe estava parasitado

Tabela 2. Relação entre as classes de tamanho do teleósteo Aluterus monoceros e as espécies de larvas de nematoides Anisakidae coletadas, indicando a prevalência (P), intensidade, intensidade média (I/IM) e abundância média de infecção (AM), nos peixes comercializados no estado do Rio de Janeiro, no período de maio a agosto de 2006.

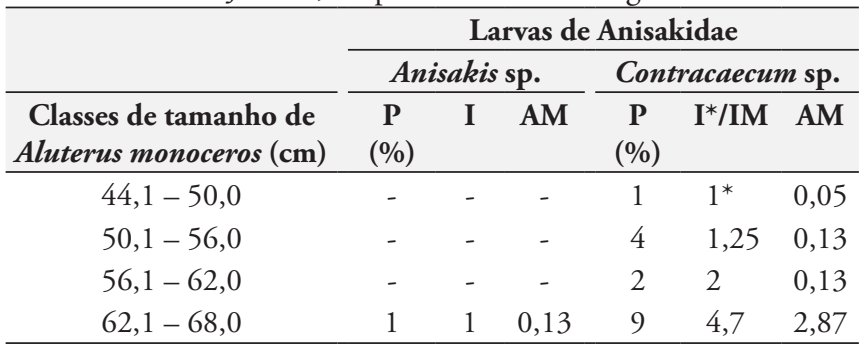

*apenas um peixe estava parasitado

Tabela 3. Relação entre as classes de tamanho do teleósteo Aluterus monoceros e as espécies de plerocercos de Trypanorhyncha coletados, indicando a prevalência (P), intensidade, intensidade média (I/IM) e abundância média de infecção (AM), nos peixes comercializados no estado do Rio de Janeiro, no período de maio a agosto de 2006.

\begin{tabular}{|c|c|c|c|c|c|c|}
\hline \multirow{3}{*}{$\begin{array}{c}\text { Classes de tamanho de } \\
\text { Aluterus monoceros }(\mathrm{cm})\end{array}$} & \multicolumn{6}{|c|}{ Plerocercos de Trypanorhyncha } \\
\hline & \multicolumn{3}{|c|}{$\begin{array}{l}\text { Floriceps } \\
\text { saccatus }\end{array}$} & \multicolumn{3}{|c|}{$\begin{array}{c}\text { Callitetrarhynchus } \\
\text { speciosus }\end{array}$} \\
\hline & $\begin{array}{c}P \\
(\%)\end{array}$ & IM & $\mathbf{A M}$ & $\begin{array}{c}\mathbf{P} \\
(\%) \\
\end{array}$ & IM & AM \\
\hline $44,1-50,0$ & 11 & 4,2 & 2,9 & 2 & 1 & 0,1 \\
\hline $50,1-56,0$ & 19 & 2,5 & 1,3 & 2 & 3 & 0,2 \\
\hline $56,1-62,0$ & 10 & 2,5 & 0,8 & 1 & $1^{*}$ & 0,03 \\
\hline $62,1-68,0$ & 5 & 1 & 1,7 & 1 & $1^{*}$ & 0,06 \\
\hline
\end{tabular}

*plerocerco coletado em um peixe

do peixe, ao local de captura, concorrência por alimento e/ou presença do hospedeiro definitivo na região, conforme mencionado por Torres, Moya e Lamilla (2000).

Comparando-se com o registro de Luque e Poulin (2004), no qual relataram o parasitismo por Contracaecum sp. em $A$. monoceros, observou-se que o tamanho médio das amostras por eles estudadas $(31,2 \mathrm{~cm}$ CT) foram inferiores à média dos peixes desta pesquisa, fato que poderia ter influenciado na carga parasitária, embora na classe $\mathrm{A}$ e $\mathrm{C}$ a prevalência tenha sido menor que a daqueles autores.

A tendência de aumento do parasitismo em relação ao comprimento do peixe permite afirmar que houve maior concentração de Contracaecum sp. $(\mathrm{p}<0,05)$, à medida do crescimento dos peixes, fato também observado por outros autores e justificado pelo parasitismo cumulativo (ALVES; LUQUE; ABDALLAH, 2003). Entretanto, devem ser evitadas generalizaçóes relacionadas à influência do tamanho do peixe sobre a composição das infracomunidades parasitárias (SAAD-FARES; COMBES, 1992)

Silva e São Clemente (2001) observaram em dourado, Coryphaenae hippurus L., filés parasitados por Floriceps saccatus, com prevalência de $6,25 \%$ e intensidade média de infecção de 1,04, índices inferiores ao presente trabalho em que a prevalência foi $45 \%$ e intensidade média de infecção de 3,17. Carbonell et al. (1998), ao pesquisar C. hippurus, proveniente do Mar Mediterrâneo e do Oceano Atlântico, nos anos de 1990 e 1991, registraram prevalência de 6,76 e 3,6\%, respectivamente. E observaram que as amostras de $C$. hippurus acima de $60 \mathrm{~cm}$ apresentaram, nos anos de 1991, 1994 e 1995, a prevalência de 28\%. Enquanto em 1990 foi encontrada a prevalência de $60 \%$, sugerindo que a correlação positiva entre o tamanho do peixe e o parasitismo esteve relacionada com a mudança de alimentação observada no estômago dos peixes estudados. Sáo Clemente et al. (1995) sugeriram que esse grupo de cestoides tem parasitismo cumulativo. Entretanto, comparando-se com os índices parasitários desta pesquisa, verifica-se que, nas duas classes de maior tamanho, $\mathrm{C}$ e $\mathrm{D}$, as prevalências foram menores, reforçando a idéia de que generalizaçóes em ecologia parasitária devem ser evitadas.

O gênero Callitetrarhynchus Pintner, 1931, descrito em ampla variedade de peixes, é constituído por duas espécies: $C$. gracilis (Rudolphi, 1819) e C. speciosus. Conforme citado por Lima (2004), os peixes Pomatomus saltator L. [=Pomatomus saltatrix (Linnaeus, 1766)], Netuma barba (Lacepède, 1803) [=Genidens barbus (Lacepède, 1803)] e Micropogonias furnieri (Desmarest, 1823) foram registrados, no litoral do Brasil, como hospedeiros de $C$. speciosus. Esse autor, pesquisando algumas espécies de garoupa, relatou o encontro desse cestoide, em Epinephelus morio (Vallenciennes, 1828), com prevalência de 5,8\% e intensidade de 2, resultado semelhante ao do presente trabalho. Em Epinephelus guaza, (Linnaeus, 1758) encontrou prevalência de 16,6\% e intensidade de 1 , sendo a prevalência superior a desta pesquisa, porém a intensidade foi menor. Em Epinephelus flavolimbatus, (Poey, 1865) verificou uma prevalência de $40 \%$, intensidade média de 6 e variando de 3 a 9; e, em Epinephelus niveatus, (Valenciennes, 1828) descobriu uma prevalência de 56,6\%, intensidade de 3,8 e variando de 1 a 18 larvas por peixe. Em ambas as espécies, os índices parasitários apresentaram-se maiores do que os encontrados neste estudo em $A$. monoceros. No peixe olho-de-cão, Priacanthus arenatus Cuvier, 1829 Lima (2004) relatou prevalência de 12,5\% e intensidade de 1 , sendo a prevalência superior e a intensidade inferior à encontrada nesta pesquisa.

Embora as larvas de anisaquídeos tenham sido encontradas no fígado e mesentério, o risco em termos de Saúde Pública não está eliminado, uma vez que existe a possibilidade de migração 
dessas larvas para a musculatura do hospedeiro, tanto no peixe vivo quanto após a captura, principalmente em razão do tempo de permanência no barco e/ou entreposto, sem evisceração, bem como a utilização das vísceras em pratos típicos.

Cabe ressaltar a importância da conscientização pelos profissionais de Vigilância e Inspeção Sanitária e dos demais relacionados com a cadeia de produção, desde a captura até o consumo, com respeito às ictioparasitoses e formas de profilaxia, implementando programas de educação sanitária em todos os níveis.

Sugere-se a aplicação do plano de Análise de Perigos e Pontos Críticos de Controle (APPCC) em todos os pontos da cadeia de produção, com a finalidade de eliminar, prevenir ou reduzir os riscos, garantindo produto com qualidade e segurança. Neste caso, com a evisceração à bordo, nos entrepostos, indústrias ou varejitas, com o devido destino do descarte.

Deve-se continuar com o desenvolvimento de pesquisas com cestoides da Ordem Trypanorhyncha para avaliar seu potencial zoonótico em relação às suas toxinas, que têm sido citadas como possíveis causadores de alergias.

\section{Referências}

ALVES, D. R.; LUQUE, J. L.; ABDALLAH, V. D. Metazoan parasites of Chub mackerel, Scomber japonicus Houttuyn (Osteichthyes: Scombridae), from the coastal zone of the state of Rio de Janeiro, Brazil. Revista Brasileira de Parasitologia Veterinária, v. 12, n. 4, p.164-170, 2003.

AUDICANA, M. et al. Clinical manifestations of allergy to Anisakis simplex. Journal of Allergy and Clinical Imunology, v. 55, supl. 59, p. 28-33, 2000.

BRASIL. Ministério da Agricultura, Pecuária e Abastecimento. Secretaria de Defesa Agropecuária. Departamento de Inspeção de Produtos de Origem Animal. Legislaçáo: RIISPOA. Brasília, 2007. 252 p.

BUSH, A. O. et al. Parasitology meets ecology on its own terms. Journal of Parasitology, v. 83, n. 4, p. 575-583, 1997.

CAMPBELL, R. A.; BEVERIDGE, I. Order Trypanorhyncha Diesing, 1863. In: KHALIL, L. F.; JONES, A.; BRAY, R. A. (Eds.). Keys to the cestodes parasites of vertebrates. Cambridge: CABI, 1994. p. 51-148.

CARBONELL, E.; CASTRO, J. J.; MASSUTI, E. Floriceps saccatus plerocerci (Trypanothyncha, Lacistorhynchidae) as parasites of dolphin fish (Coryphaena hippurus L.) and pompano dolphin (Coryphaena equiselis L.) in western mediterranean and eastern atlantic waters: ecological and biological aspects. Journal of Parasitology, v. 84, n. 5, p. 1035-1039, 1998.

CARVAJAL, J.; REGO, A. A. Critical studies on the genus Callitetrarhynchus (Cestoda: Trypanorhyncha) with recognition of Rhynchobothrium speciosum Linton, 1897 as a valid species of the genus Callitetharhynchus. Systematic Parasitology, v. 7, n. 3, p. 161-167, 1985.

DEARDORFF, T. L.; RAYBOURNE, R. B.; MATTIS, T. E. Infections with Trypanorhyncha plerocerci (Cestoda) in Hawaiian fishes of commercial importance. Quarterly University of Hawaiian Sea Grant College Program, v. 6, n. 3, p. 1-6, 1984.

DOLLFUS, R. P. H. Études critiques sur les tetrarhynches du Museúm de Paris. Arquives du Museúm National d'Histoire Naturelle, v. 19, p. 1-466, 1942.
EIRAS, J. C.; TAKEMOTO, R. M.; PAVANELLI, G. C. Métodos de estudos e técnicas laboratoriais em parasitologia de peixes. 2. ed. Maringá: EDUEM, 2006. 199 p.

FERRE, I. Anisakiosis y otras zoonosis parasitarias transmitidas por consumo de pescado. Revista AquaTIC, v. 14, n. 6, 2001. Disponível em: <http://www.revistaaquatic.com/>. Acesso em: 12 de dezembro de 2006.

FIGUEIREDO, J. L.; MENEZES, N. A. Manual de peixes marinhos do Sudeste do Brasil. São Paulo: Museu de Zoologia - USP, 2000. 116 p.

GERMANO, P. M. L.; GERMANO, M. I. S. Anisaquíase: zoonose parasitária emergente no Brasil? Higiene Alimentar, v. 12, n. 54, p. 26-35, 1998 .

GONZÁLEZ, I. D. Anisakis en el pescado: prevención y control: seguridade alimentaria y alimentación weblog gestionado por el Programa Vigilância Sanitária. Disponível em: <http://weblogs.madrimasd.org/ alimentacion/archive/2006/07/11/34816.aspx-55k>. Acesso em: $14 \mathrm{de}$ dezembro de 2006.

KNOFF, M. et al. Anisakidae parasitos de congro-rosa, Genypterus brasiliensis Regan, 1903 comercializados no estado do Rio de Janeiro, Brasil de interesse na saúde pública. Parasitologia Latinoamericana, v. 62, n. 3-4; p. 127-133, 2007.

LIMA, F. C. Cestóides da ordem Trypanorhyncha em peixes teleósteos comercializados no estado do Rio de Janeiro. 2004. 83 f. Tese (Doutorado) - Universidade Federal Fluminense, Niterói, 2004.

LUQUE, J. L.; POULIN, R. Use of fish a intermediate hosts by helminth parasites: a comparative analysis. Acta Parasitologica, v. 49, n. 4, p. 353-361, 2004.

PETTER, A. J.; MAILLARD, C. Larves d'ascarides parasites de poissons en Méditerranée occidentale. Bulletin du Museúm National d'Histoire Naturelle, v. 10, n. 2, p. 347-369, 1988

REGO, A. A. et al. Parasitas de anchovas, Pomatomus saltatrix (L.) do Rio de Janeiro. Ciência e Cultura, v. 35, n. 9, p. 1329-1336, 1983.

RODERO, M.; CUÉLLAR, C. Humoral immune responses induced by Gymnorhynchus gigas extracts in BALB/ c mice. Journal of Helminthology, v. 73 , p. $239-243,1999$.

SAAD-FARES, A.; COMBES, C. Abundance/host size relationship in a fish trematode community. Journal of Helminthology, v. 66, p. $187-192,1992$.

SÃO CLEMENTE, S. C. et AL. Cestóides Trypanorhyncha parasitos de peixe sapo-pescador, Lophius gastrophysus Miranda Ribeiro, 1915 comercializados no estado do Rio de Janeiro, Brasil. Revista Brasileira de Parasitologia Veterinária, v. 16, n. 1, p. 37-42, 2007.

SÃO ClEMENTE, S. C.; LIMA, F. C.; UCHOA, C. M. A. Parasitos de Balistes vetula (L.) e sua importância na inspeção do pescado. Revista Brasileira de Ciência Veterinária, v. 2, n. 2, p. 39-41, 1995.

SILVA, C. M.; SÃO CLEMENTE, S. C. Nematóides da família Anisakidae e cestóides da Ordem Trypanorhyncha em filés de dourado (Coryphaena hippurus) e ariocó (Lutjanus synagris) e sua importância na inspeção de pescado. Higiene Alimentar, n. 15, n. 80-81, p. 75-79, 2001.

TORRES, P.; MOYA, R.; LAMILLA, J. Nematodos anisákidos de interés en salud pública em peces comercializados em Valdivia, Chile. Archivos de Medicina Veterinária, v. 32, n. 1, p. 107-113, 2000.

VÁZQUEZ-LÓPEZ, C et al. A 24-kDa collagenase from Gymnorhynchus gigas elicts rat ileum hyperreactivity and is a target of humoral responses in mice previously given a single oral dose of parasite extract. Digestive Diseases and Sciences, v. 47, n. 4, p. 935-942, 2002. 